\title{
Transcranial Magnetic Stimulation Reveals Two Cortical Pathways for Visual Body Processing
}

\author{
Cosimo Urgesi, ${ }^{1}$ Beatriz Calvo-Merino, ${ }^{2}$ Patrick Haggard, ${ }^{2}$ and Salvatore M. Aglioti ${ }^{3,4}$ \\ ${ }^{1}$ Istituto di Ricovero e Cura a Carattere Scientifico (IRCCS) “E. Medea," Polo Friuli Venezia Giulia, I-33078 San Vito al Tagliamento, Pordenone, Italy, \\ ${ }^{2}$ Institute of Cognitive Neuroscience and Psychology Department, University College London, London WC1N 3AR, United Kingdom, ${ }^{3}$ Dipartimento di \\ Psicologia, Università di Roma “La Sapienza," I-00185 Rome, Italy, and ${ }^{4}$ IRCCS Fondazione Santa Lucia, I-00179 Rome, Italy
}

Visual recognition of human bodies is more difficult for upside down than upright presentations. This body inversion effect implies that body perception relies on configural rather than local processing. Although neuroimaging studies indicate that the visual processing of human bodies engages a large fronto-temporo-parietal network, information about the neural underpinnings of configural body processing is meager. Here, we used repetitive transcranial magnetic stimulation (rTMS) to study the causal role of premotor, visual, and parietal areas in configural processing of human bodies. Eighteen participants performed a delayed matching-to-sample task with upright or inverted static body postures. Event-related, dual-pulse rTMS was applied $150 \mathrm{~ms}$ after the sample stimulus onset, over left ventral premotor cortex (vPMc), right extrastriate body area (EBA), and right superior parietal lobe (SPL) and, as a control site, over the right primary visual cortex (V1). Interfering stimulation of vPMc significantly reduced accuracy of matching judgments for upright bodies. In contrast, EBA rTMS significantly reduced accuracy for inverted but not for upright bodies. Furthermore, a significant body inversion effect was observed after interfering stimulation of EBA and V1 but not of vPMc and SPL. These results demonstrate an active contribution of the fronto-parietal mirror network to configural processing of bodies and suggest a novel, embodied aspect of visual perception. In contrast, the local processing of the body, possibly based on the form of individual body parts instead of on the whole body unit, appears to depend on EBA. Therefore, we propose two distinct cortical routes for the visual processing of human bodies.

Key words: body perception; body inversion effect; extrastriate body area; premotor cortex; mirror neurons; repetitive transcranial magnetic stimulation

\section{Introduction}

The human brain has an inherent ability to process information about human bodies, which presumably reflects the primary importance of this class of stimuli for our social life. The "inversion effect" refers to the better visual recognition of specific classes of objects when presented in upright than upside down position. This effect, originally reported for faces (Yin, 1969), has also been found for bodies (Reed et al., 2003), and it is seen as an indicator of configural processing (Maurer et al., 2002; Reed et al., 2006a). However, the neural bases of configural processing for body stimuli are still primarily undefined. Here, we investigated how human bodies are processed in different visual, premotor, and parietal body-related areas.

Visual processing of human bodies involves selective activations of a lateral occipito-temporal area, called extrastriate body area (EBA) (Downing et al., 2001). This area responds to the

Received Feb. 21, 2007; revised May 17, 2007; accepted June 13, 2007.

This work was supported by the Royal Society European Science Exchange Programme (P.H.) and the Ministero Italiano Università e Ricerca and Fondo Investimenti per la Ricerca di Base (S.M.A.). B.C.-M. was supported by a grant from the Leverhulme Trust to P.H. We are grateful to Tom Sapsford and Mavin Khoo for their help in designing the stimuli.

Correspondence should be addressed to either Dr. Salvatore M. Aglioti or Dr. Cosimo Urgesi, Department of Psychology, University of Rome "La Sapienza," Via dei Marsi 78, I-00185 Rome, Italy. E-mail: salvatoremaria.aglioti@uniroma1.it or cosimo.urgesi@uniud.it.

DOI:10.1523/JNEUROSCI.0789-07.2007

Copyright $\odot 2007$ Society for Neuroscience $\quad$ 0270-6474/07/278023-08\$15.00/0 visual presentation not only of the whole body but also of its single parts (Downing et al., 2001). Yet, recent studies have shown body-selective responses in the posterior temporal cortex that are reduced by body inversion (Stekelenburg and de Gelder, 2004; Thompson et al., 2005). One may thus wonder whether EBA may be specifically involved in configural processing of bodies as a complementary counterpart of the fusiform face area (Kanwisher et al., 1997), which plays an important role in the configural processing of faces (Yovel and Kanwisher, 2005). Perception of bodies and faces, however, may involve different kinds of configural processing (Reed et al., 2006a). Indeed, because the relative position of body-part changes during action, body perception requires a fine-grained structural description of the spatial relationships among moving body parts (Reed et al., 2006a). Viewing body movements engages a fronto-parietal network that matches action observation with action execution (mirror neuron system) (Rizzolatti and Craighero, 2004). Furthermore, mental transformation of body in space selectively activates superior parietal lobe (SPL) (Bonda et al., 1995). Recent repetitive transcranial magnetic stimulation (rTMS) studies have shown that the ventral premotor cortex (vPMc) plays a crucial role in the visual discrimination of body actions (Pobric and Hamilton, 2006; Urgesi et al., 2007). What remains unclear is whether the body processing in premotor and parietal areas involves overall configurations, or local details of human bodies. 
Here, we used event-related rTMS to create temporary inactivation of striate and extrastriate visual cortex and premotor and parietal areas during visual processing of upright and inverted body postures. Although neuroimaging studies cannot establish whether activity in these areas is crucial to body processing or merely epiphenomenal, using rTMS allowed us to explore the causal link between neural activity and specific neural processes putatively involved in body perception. By using the body inversion effect as a measure of configural processing, we predicted the fate of upright and inverted postures perception after rTMS inactivation of areas supposedly belonging to the configural or to local processing systems. Normal perception of upright bodies may rely both on a configural system for processing whole-body shape and also a local system for processing body-part details. Body inversion disrupts configural processing (Reed et al., 2006a). Therefore, we pre-

dicted that rTMS interference with configural processing areas should selectively disrupt processing of upright bodies. In contrast, interference with local processing areas should disrupt processing of inverted bodies (Fig. 1).

\section{Materials and Methods}

Participants. Eighteen healthy individuals ( 16 women) aged $20-30$ years (mean, 24.4 years) were recruited for the study. All participants were right handed according to a standard handedness inventory (Briggs and Nebes, 1975). They had normal or corrected-to-normal visual acuity in both eyes and were naive as to the purposes of the experiment. Participants gave their written informed consent and were paid 15 Euros for their participation in the study. The procedures were approved by the ethics committee of the Fondazione Santa Lucia (Rome, Italy) and were in accordance with the ethical standards of the 1964 Declaration of Helsinki. None of the participants had neurological, psychiatric, or other medical problems or any contraindication to rTMS (Wasserman, 1998). No discomfort or adverse effects during rTMS were reported or noticed.

Stimuli and task. Experimental stimuli were color pictures of body postures taken with a digital camera in front of a neutral chromablue background. Postures involved different lower- and upper-limb configurations of the left and right side of the body (Fig. $2 \mathrm{~A}$ ). The body stimuli were chosen to be similar to those previously shown to activate EBA (Downing et al., 2001). The postures involved complex configurations of body parts in space, which might be expected to activate SPL (Bonda et al., 1995; Buccino et al., 2001; Calvo-Merino et al., 2005). In addition, although static images of body postures were used, the stimuli clearly showed the body in a range of different, noncanonical postures. Participants performed the matching-to-sample task schematically represented in Figure $2 \mathrm{~B}$. On each trial, participants were presented with a single, 100 ms lasting body posture, followed by a 500 ms mask. On mask disappearance, a stimulus showing two postures was presented. Subjects were asked to decide, as accurately and fast as possible, which stimulus in the pair matched the previously seen single sample. In half of the trials, the sample and the probe stimuli were shown in their canonical upright position, whereas they were inverted in the other half of the trials. In keeping with our previous studies (Urgesi et al., 2004, 2007), the rTMS train was triggered by the presentation of the sample stimulus. Therefore, rTMS was supposed to interfere with perceptual processing rather than with response programming, which occurred much later. Studies have shown that rTMS may induce site-independent, nonspecific effects on manual responses [e.g., shortening of response latencies (Terao et al.,
1997; Sawaki et al., 1999)] that may mask site-specific modulations of performance. Our matching-to-sample paradigm aimed to separate the phase of stimuli perception from the response phase, thus ruling out any confound of nonspecific rTMS effect on manual responses. The temporal relationship between rTMS trains and presentation of the sample stimulus was designed to interfere with its perception. Indeed, the first pulse was delivered $150 \mathrm{~ms}$ after the sample onset, a stimulation timing that is thought to interfere with perceptual processing in extrastriate (Amassian et al., 2002; Urgesi et al., 2004) and premotor (Urgesi et al., 2007) cortex. One may note that our task required both perceptual processing and memory of the sample stimulus and that rTMS may have affected the maintenance of the sample stimulus in visual working memory. Although this possibility cannot be excluded, it should be noted that rTMS effects on memory functioning have been reported with longer and more delayed trains (Oliveri et al., 2001; Postle et al., 2006; Luber et al., 2007). Moreover, maintenance in visual working memory seems to be supported by activations of inferior and middle temporal areas that are involved in the visual processing of the same stimuli (Ranganath and D'Esposito, 2005).

Twenty single samples were used. Half of the single samples reproduced standard body positions selected from the classical ballet-style repertoire; the other half was obtained by combining the upper or the lower part of each classical ballet posture with that of a comparable posture of a different dance-style repertoire. During the matching phase, the sample stimulus was presented along with a distractor posture that differed in upper-limb configuration in half of the trials and in lowerlimb configuration in the other half. This allowed us to control and balance the body-part differences on which the discrimination task is supposedly based. Thus, 20 pairs of postures were used for the matching phase (examples of the pairs are shown in Fig. 2 A). For each pair, one of the postures was a standard dance posture, and the other was a combined dance-like posture. We balanced across pairs the presentation of the original classic ballet postures or of the combined postures as sample stimuli. None of participants had extensive experience with dance or knew the names of the dance postures. Moreover, half of the stimuli were nonstandard adaptations of dance postures, specifically to discourage use of verbal/conceptual knowledge in the match-to-sample task. In view of this, it is highly unlikely that the combined postures used in our study were semantically categorized by the participants. All of the postures were performed by a male actor with experience in dance movements. The actor wore a fit black suit during the photo session to emphasize the appearance of body configurations. For each pair, the stimuli were matched for perspective and luminance. The color pictures were edited 
A
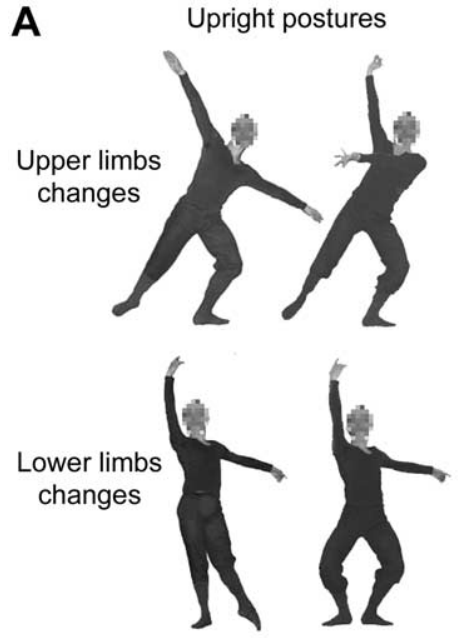

B

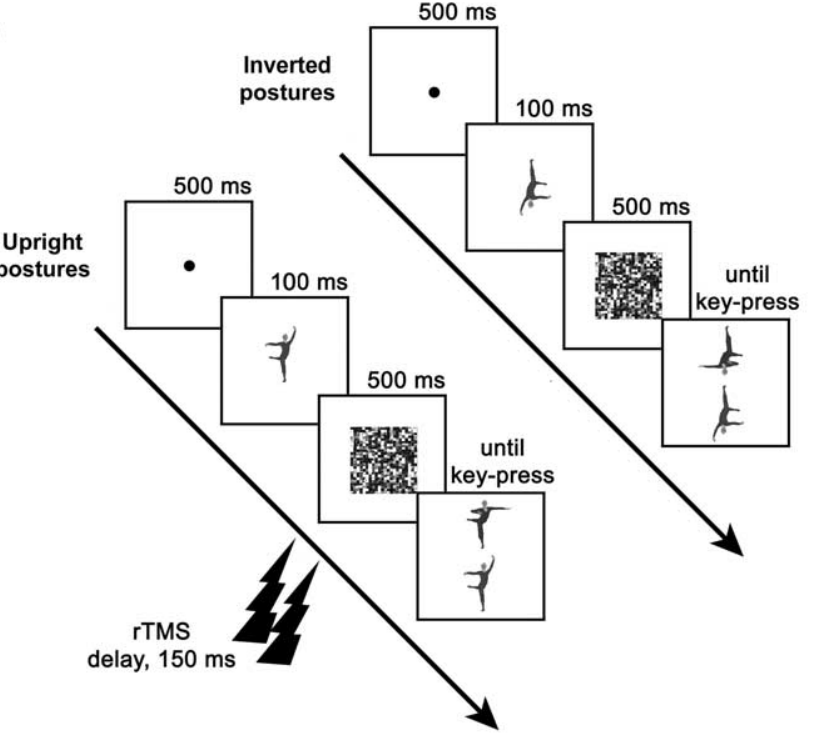

Figure 2. Examples of stimuli and timeline of the experiment. $A$, Examples of upright and inverted pairs of stimuli in which upper-limb (top row) or lower-limb (bottom row) configuration is changed compared with the original posture (leftmost images). $\boldsymbol{B}$, The typical sequence of a trial is shown for the matching-to-sample task with upright and inverted postures. A two-pulse train of rTMS was applied $150 \mathrm{~ms}$ after the onset of the sample stimulus.

by means of the Adobe Photoshop software (Adobe Systems, San Jose, CA). The background was removed, and the actor's face was blurred to ensure that discrimination performance relied on body rather than facial cues. To create the inverted stimuli, the original stimuli were rotated $180^{\circ}$. Thus, experimental stimuli consisted of 20 pairs of upright and 20 pairs of inverted postures.

TMS. Participants wore a tightly fitting bathing cap on which the stimulation points of the scalp were marked. For each participant, the resting motor threshold for the first dorsal interosseous muscle of the right hand was determined. Surface $\mathrm{Ag} / \mathrm{AgCl}$ electrodes were placed in a belly-tendon montage. An electromyographic signal was amplified at a gain of $1000 \times$ by a Digitimer (Hertfordshire, UK) D360 amplifier, bandpass filtered $(20 \mathrm{~Hz}$ to $2.5 \mathrm{kHz}$ ), and digitized (sampling rate, $5 \mathrm{kHz}$ ) by means of a CED Power 1401 controlled with Spike 2 software (Cambridge Electronic Design, Cambridge, UK). The resting motor threshold, defined as the lowest intensity able to evoke 5 of 10 motor-evoked potentials with an amplitude of at least $50 \mu \mathrm{V}$, was determined by holding the stimulation coil over optimal scalp position (i.e., the motor cortex area controlateral to the right hand producing the largest potentials) for the first dorsal interosseous muscle.

Stimulation sites were identified on each participant's scalp with the
SofTaxic Navigator system (EMS, Bologna, Italy). In keeping with the hemispheric dominance previously reported in studies of premotor function (Rizzolatti and Craighero, 2004) and of EBA (Downing et al., 2001), we delivered rTMS over the left vPMc, right EBA, and right SPL. Coordinates in Talairach space (Talairach and Tournoux, 1988) were automatically estimated by the SofTaxic Navigator from a magnetic resonance imaging-constructed stereotaxic template. The scalp locations that corresponded best to left vPMc and to right EBA and SPL coordinates (Fig. 3) were identified and marked with a pen. Moreover, an occipital site $2 \mathrm{~cm}$ above and $2 \mathrm{~cm}$ lateral to the inion on the right, corresponding to primary visual cortex (V1), was targeted to control for nonspecific effects of rTMS on visual perception.

rTMS was performed by connecting two Magstim Model 200 stimulators with Bistim module (The Magstim Company, Carmarthenshire, Wales, UK), producing a maximum output of $1.75 \mathrm{~T}$ at the coil surface (stimulus attenuation, 22\%; duration, $1 \mathrm{~ms}$; rise time, $110 \mu \mathrm{s}$ ). Two pulses were delivered with an interstimulus interval of $100 \mathrm{~ms}$ by means of a $70 \mathrm{~mm}$ figure-eight stimulation coil (Magstim polyhurethane-coated coil). In keeping with previous studies showing an rTMS-suppressive effect on EBA and vPMc (Urgesi et al., 2004, 2007), the first rTMS pulse was delivered $150 \mathrm{~ms}$ after the onset of sample presentation. At this time interval, the stimulation of $\mathrm{V} 1$ is generally ineffective on visual tasks (Amassian et al., 2002); thus, V1 stimulation served as the control site for the nonspecific effect of rTMS per se. The same pulse delay and stimulation intensity was used for the four stimulation sites. Stimulation intensity was $120 \%$ of the resting motor threshold for both pulses and ranged from 37 to $65 \%$ (mean, 51.7\%) of the maximum stimulator output. During stimulation of the four sites, the coil was held by hand tangential to the scalp, with the handle pointing backward and medially at a $45^{\circ}$ angle from the middle sagittal axis of the participants' head. The position of the coil with respect to the marks was checked continuously. During stimulation, participants wore commercial earplugs to protect their hearing. None of the participants reported phosphenes or muscular twitches after rTMS of any cortical site.

Procedure. Each participant was tested in a single experimental session lasting $\sim 2 \mathrm{~h}$. Participants completed a 20 -trial practice block before proceeding to the experimental blocks. During the experimental session, two blocks of 20 trials were presented for each stimulation site. For each participant, each of the four stimulation conditions was repeated twice according to an across-subjects counterbalanced sequence. In each block, 10 upright and 10 inverted stimuli were randomly presented, for a total of 20 upright and 20 inverted trials per stimulation site. A short rest was allowed before proceeding to a different block. Participants sat $57 \mathrm{~cm}$ away from a 17 inch monitor (resolution, $1024 \times 768$ pixels; refresh frequency, $99 \mathrm{~Hz}$ ), where stimuli appeared on a white background and subtended a $8 \times 9.1^{\circ}$ square region around the fovea. Stimuluspresentation timing, rTMS triggering, and randomization were controlled by a custom software created using Matlab (MathWorks, Natick, MA) and the Psychophysics Toolbox extensions. During the experiment, all participants had their chin restrained and their head aligned with the center of the viewing screen. Eye position was monitored, and fixation was checked continuously during tachistoscopic presentation by means of a rear view mirror. A trial started with the presentation of a central fixation point lasting $500 \mathrm{~ms}$. The sample stimulus was presented for 100 $\mathrm{ms}$ at the center of the monitor. Image persistence was limited by presenting a random-dot mask $\left(9.1 \times 9.1^{\circ}\right.$ in size; duration, $\left.500 \mathrm{~ms}\right)$. During mask presentation, a dual-pulse rTMS train was delivered $150 \mathrm{~ms}$ after the onset of the sample stimulus. Immediately after the disappearance of the mask, the two probe stimuli appeared and remained on the screen until a response was made. Participants were asked to respond by using their right index or middle finger to press the left or the right key, respectively, on a custom-made response box. Each key corresponded to one of the two positions on the screen on which the probe stimuli were presented; the position of the probe stimuli was randomized in each trial. Because in a previous study (Urgesi et al., 2007) the interfering effects of stimulation of EBA and vPMc in the left or in the right hemisphere were independent from the responding hand, all participants used their right hand. Reaction times (RTs) and accuracy were automatically recorded and stored for analysis. 
After the rTMS session, each single posture used in the experimental sessions was presented in a counterbalanced order on the computer monitor. Each participant was asked to judge the perceived amount of the motion implied in each image on a 7-point Likert-like scale, with 1 indicating "no effect" and 7 indicating "maximal effect imaginable."

Data handling. For each stimulation site, we computed individual mean percentages of correct responses for the upright and the inverted postures as an index of accuracy (20 trials per cell). Moreover, RTs to correct responses provided a speed index. To directly compare the site-specific effect of rTMS on body inversion effect and to protect us from capitalizing on significance threshold, we computed the ratio between matching accuracy for inverted and upright postures (body inversion effect ratio) for each target site stimulation. Body inversion
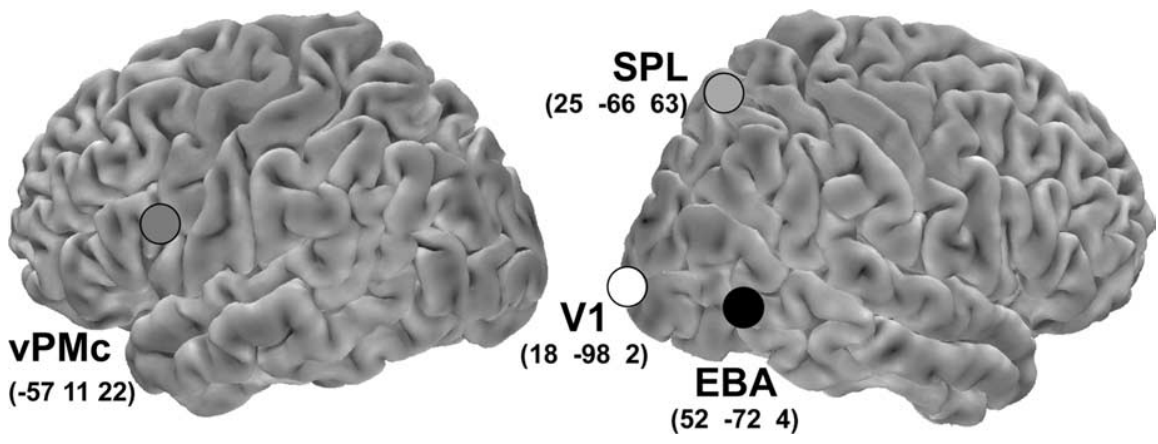

Figure 3. Stimulation sites plotted on the lateral views of a standard brain. Mean Talairach coordinates of the stimulation sites were as follows: $x=-57, y=11, z=22$ for left vPMc, corresponding to Brodmann's area 44 in the pars opercularis of the left inferior frontal gyrus; $x=52, y=-72, z=4$ for right EBA, corresponding to Brodmann's area 37 in the posterior part of the right middle temporal gyrus; $x=25, y=-66, z=63$ for the right SPL, corresponding to Brodmann's area 7 in the posterior part of the right superior parietal lobule; $x=18, y=-98, z=2$ for the V1, corresponding to Brodmann's area 17 , in the right middle occipital gyrus. effect ratios for EBA, SPL, and vPMc rTMS were then normalized with respect to the body inversion effect index for the V1 control stimulation site (e.g., [target area - control area]/[target area + control area]). This procedure allowed us to directly test the effect of rTMS independently of the individual differences in the absolute level of body inversion effect. Data were submitted to separate repeatedmeasures ANOVA, with a significance threshold of $p<0.05$. Post hoc multiple comparisons were performed using the Duncan test.

\section{Results}

In the present study, we used rTMS to create temporary inactivation of specific cortical areas during visual body processing, an approach that allowed us to explore the causal influence of neural activity in these areas on the type of processing strategy adopted for body perception. We presented upright and inverted body postures while stimulating striate and extrastriate visual cortex and premotor and parietal areas. We compared performances for accuracy and RTs for upright and inverted postures after stimulation of the four target sites. Although rTMS may not reach body-selective fusiform areas (Peelen and Downing, 2005a), this technique is particularly appropriate to interfere with neural activity of areas located on the lateral surface of the cortex. Therefore, we targeted three body-related lateral areas and tested their role in configural or local body processing. We predicted that interfering with configural or local systems should impair upright or inverted body postures, respectively. In particular, interference with the configural system is expected to disrupt processing of upright postures, while leaving intact the perception of inverted postures. In contrast, interference with the local system should disrupt local processing of body-part details in the inverted postures, while leaving intact the perception of upright postures.

A two-way repeated-measures ANOVA was performed on accuracy, with orientation (upright, inverted postures) and stimulation site (V1, EBA, SPL, vPMc) as within-subjects variables (Fig. 4A). The main effect of orientation was significant $\left(F_{(1,17)}=\right.$ $\left.16.26 ; p=0.001 ; \eta_{\mathrm{p}}{ }^{2}=0.489\right)$, because observers were more accurate for matching upright $(87.71 \pm 1.5 \%)$ than inverted $(82.92 \pm 1.54 \%)$ postures. This result indicates that the reduction in matching accuracy for inverted bodies in our study is comparable to that found in previous reports (Reed et al., 2003). The main effect of stimulation site failed to reach significance $\left(F_{(3,51)}\right.$ $\left.=1.87 ; p=0.146 ; \eta_{\mathrm{p}}{ }^{2}=0.099\right)$. Crucially, however, a highly significant orientation by stimulation site interaction was found $\left(F_{(3,51)}=4.7 ; p=0.006 ; \eta_{\mathrm{p}}{ }^{2}=0.216\right)$. Post hoc comparisons
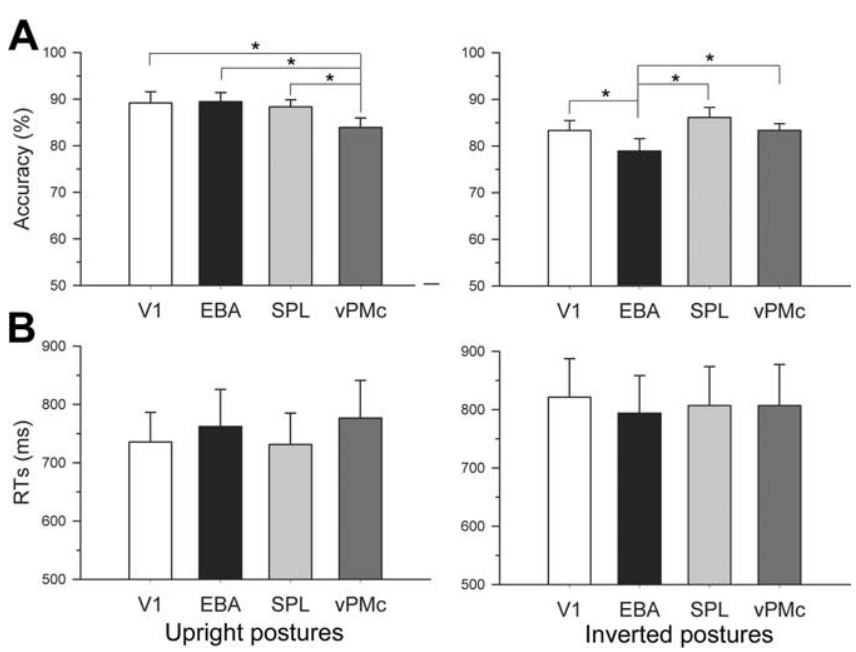

Figure 4. Mean ( \pm SEM) accuracy and RT results. A, Accuracy for matching upright postures was impaired after stimulation of left vPMc compared with stimulation of V1, EBA, and SPL. In contrast, accuracy for matching inverted postures was impaired after stimulation of right EBA. $B$, No effect of stimulation was observed on RTs. ${ }^{*} p<0.05$.

showed that matching upright postures was significantly impaired after vPMc rTMS $(83.89 \pm 2.04 \%)$ compared with stimulation of EBA $(89.44 \pm 1.93 \% ; p=0.017), \mathrm{V} 1(89.17 \pm 2.37 \% ; p$ $=0.02)$, and SPL $(88.33 \pm 1.51 \% ; p=0.044)$. No difference was observed between stimulation of EBA and stimulation of V1 ( $p$ $=0.893)$ and SPL $(p=0.613)$ as well as between stimulation of $\mathrm{V} 1$ and stimulation of SPL $(p=0.685)$. In striking contrast, matching inverted postures was significantly impaired after EBA rTMS $(78.89 \pm 2.67 \%)$ compared with stimulation of V1 $(83.33 \pm 2.1 \% ; p=0.044)$, SPL $(86.11 \pm 2.16 \% ; p=0.002)$, and vPMc $(83.33 \pm 1.46 \% ; p=0.034)$. No difference was observed between stimulation of VPMc and stimulation of V1 $(p=1)$ and SPL $(p=0.223)$ as well as between stimulation of V1 and stimulation of SPL ( $p=0.206)$. Comparing discrimination accuracy for upright and inverted postures across stimulation sites revealed that upright body postures were matched more accurately than inverted postures after stimulation of V1 $(p=0.012)$ and EBA $(p<0.001)$, but not after stimulation of SPL $(p=0.282)$ and vPMc $(p=0.801)$. Thus, the results show that stimulation of vPMc disrupted configural processing of upright bodies, while leaving intact the local processing of inverted postures. Accuracy 
A
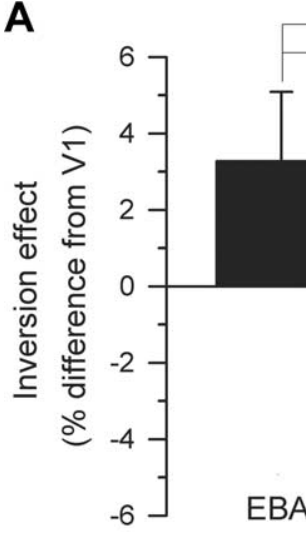

EBA
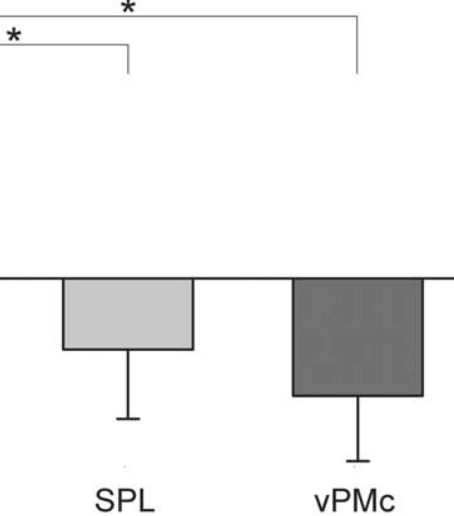

Stimulation site

B

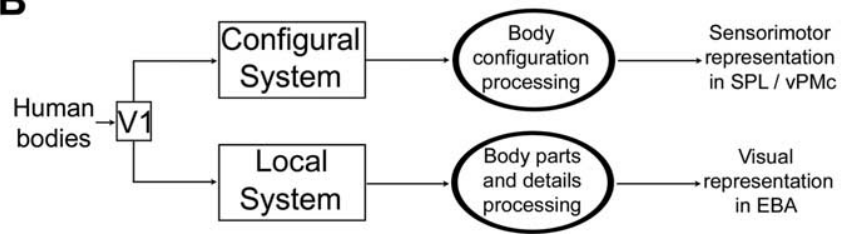

Figure 5. Two specialized systems for body perception. $A$, For each stimulated area, we computed inverted/upright postures accuracy ratios as indicators of body inversion effect. To test the effect of specific cortical virtual lesions on the body inversion effect, inversion ratios after stimulation of EBA, SPL, and VPMc were normalized to the ratio after control stimulation of V1. The normalized body inversion effect ratio was significantly higher after interfering stimulation of right EBA than of right SPL and left vPMc. Error bars indicate SEM. ${ }^{*} p<0.05$. B, Body postures seem to be processed by two partially independent cortical systems: the configural system analyzes body configurations, whereas a local system analyzes body-part details and form features. The present study suggests that configural processing may rely on sensorimotor regions, whereas local processing may rely on visual areas.

in processing upright postures after vPMc rTMS was not significantly different from processing of inverted postures after stimulation of V1 ( $p=0.787)$ and SPL ( $p=0.282)$. This shows that the interference effect of vPMc rTMS was so profound to abolish the advantage of processing upright versus inverted bodies. Therefore, vPMc interference had a specific and strong effect on configural processing. In contrast, stimulation of EBA disrupted local processing of inverted bodies while leaving intact the configural processing of upright postures. Matching inverted postures after stimulation of EBA was significantly less accurate than matching upright postures not only after stimulation of $\mathrm{V} 1(p<$ $0.001)$ and SPL $(p<0.001)$ but also after stimulation of vPMc $(p=0.028)$. This result may derive from two additive effects, namely the inhibition of areas purportedly involved in local processing and the inherent difficulty in using configural strategies when processing inverted bodies. This speaks in favor of the specific influence of EBA on local processing. In summary, we show a double dissociation of vPMc and EBA in configural and local processing of human bodies.

We further investigated the "virtual lesion" effect of areas supposedly involved in perceptuo-motor analysis of human bodies. We compared changes of body inversion effect induced by interference with cortical structures supposedly involved in local (EBA) or configural (SPL, vPMc) body processing. We first generated a single quantity to estimate the inversion effect, by taking the ratio of accuracy for inverted postures to upright postures at each site. We then normalized these ratio estimates for EBA, SPL, and $\mathrm{vPMc}$ to the same ratio after V1 stimulation (Fig. 5A). This allowed us to directly test the effect of rTMS independently from individual differences in the absolute level of body inversion effect. Figure $5 A$ suggests that body inversion effect after stimulation of EBA was significantly higher than after stimulation of SPL and vPMc. A one-way repeated-measures ANOVA with stimulation site as the within-subjects variable confirmed this suggestion by showing that rTMS caused a site-specific modulation of body inversion effect $\left(F_{(2,34)}=5.89 ; p=0.006 ; \eta_{\mathrm{p}}{ }^{2}=0.257\right)$. Indeed, the effect of EBA rTMS on the body inversion effect (3.27 \pm $1.82 \%)$ was significantly higher than the effect of SPL $(-1.86 \pm$ $1.81 \% ; p=0.013)$ and vPMc rTMS $(-3.08 \pm 1.71 \% ; p=0.004)$, which in turn did not differ from one another $(p=0.541)$. These results demonstrate site-specific modulations of body inversion effect after interference with EBA and with sensorimotor, frontoparietal sites. Furthermore, a one-sample $t$ test (one-tailed) demonstrated that the increase of body inversion effect after stimulation of EBA was significantly higher than zero $\left(t_{(17)}=1.8 ; p=\right.$ $0.045)$. In contrast, the reduction in body inversion effect was significantly lower than zero after stimulation of $\operatorname{vPMc}\left(t_{(17)}=\right.$ $-1.8 ; p=0.045)$ but not after stimulation of SPL $\left(t_{(17)}=-1.03\right.$; $p=0.16)$. Thus, body inversion effect increased after stimulation of EBA and decreased after stimulation of vPMc.

To test the effect of rTMS on latency of responses, we entered mean RTs in a two-way repeated-measures ANOVA with orientation and stimulation site as within-subjects variables (Fig. $4 B$ ). The main effect of orientation was significant $\left(F_{(1,17)}=15.1 ; p=\right.$ $\left.0.001 ; \eta_{\mathrm{p}}{ }^{2}=0.47\right)$, because RTs were lower for matching upright $(751.3 \pm 55.56 \mathrm{~ms})$ than inverted $(807.25 \pm 65.1 \mathrm{~ms})$ postures. Thus, a stable and reliable body inversion effect was obtained for both the accuracy and the RT data. The main effect of stimulation site $\left(F_{(3,51)}<1 ; \eta_{\mathrm{p}}{ }^{2}=0.018\right)$ and the interaction orientation by stimulation site $\left(F_{(3,51)}=1.9 ; p=0.142 ; \eta_{\mathrm{p}}{ }^{2}=0.1\right)$ failed to reach significance, ruling out any speed-accuracy tradeoff. Therefore, stimulation of vPMc and EBA disrupted discrimination accuracy for body perception, without affecting latency of responses. It is possible to conceive that the site-specific modulation of error rate by rTMS may have masked a possible interfering effect of rTMS on latencies of response.

Previous studies reported that the motor system may be involved in the processing of the motion implied in static images of the human bodies (Urgesi et al., 2006). To rule out that experimental effects may be accounted for by differences in the implied motion suggested by upright and inverted postures, we collected subjective reports of the perceived intensity of implied motion in the experimental stimuli. After the rTMS session, participants were required to judge on a 7-point Likert-like scale the perceived amount of the motion implied in the upright and inverted body postures stimuli. Individual mean intensity scores of implied motion for upright and inverted postures were compared by means of a $t$ test for dependent samples (two-tailed). A comparable level of implied motion perception was suggested by upright (3.97 \pm $0.27)$ and inverted $\left(4.43 \pm 0.27 ; t_{(17)}=-1.41 ; p=0.178\right)$ postures. This shows that the differential modulation of upright and inverted body perception after stimulation of EBA and vPMc was arguably attributable to the representation of the configurations or of body-part details in the different postures, rather than the degree of implied motion.

\section{Discussion}

The present study investigated the specific properties of body processing in brain areas that respond selectively to visual presentation of human bodies. We applied event-related rTMS in striate and extrastriate visual areas and in premotor and parietal areas during visual discriminations of upright and inverted body 
postures. Stimulation of left vPMc selectively impaired discrimination of upright body postures but not of inverted body postures. This result suggests that $\mathrm{vPMc}$ participates in the configural visual processing of upright bodies but not in the local processing of body-part details. Conversely, EBA rTMS selectively impaired discrimination of inverted postures but did not affect discrimination of upright postures, suggesting that EBA processes bodies according to local rather than configural strategies. Behavioral studies reported that inversion disproportionately impairs the perception of static (Reed et al., 2003) and dynamic (Dittrich et al., 1996; Pavlova and Sokolov, 2000; Clarke et al., 2005) displays of human bodies compared with other objects, thus suggesting a configural processing of human bodies. Here, we tested the possible causative role of visual and sensorimotor neural representations during visual analysis of human body stimuli. We show that rTMS of vPMc but not of EBA disrupted the body inversion effect, thus suggesting that although the former area is crucially involved in the configural processing of human bodies, the latter appears to be involved in the local processing of the body-part details.

\section{Sensorimotor processing of human bodies}

Mere observation of body actions activates the vPMc (Buccino et al., 2001; Grezes et al., 2003; Calvo-Merino et al., 2005), suggesting its involvement in the resonant process though which seen actions are mapped onto the observer's motor system (Rizzolatti and Craighero, 2004). The motor representations of observed actions may feed back into visual areas and affect perceptual processing by providing the visual system with a fine-grained description of the moving body (Wilson and Knoblich, 2005). Indeed, the process of monitoring our own body postures and actions interacts with and facilitates the representation of postures and actions of the other people's bodies (Reed and Farah, 1995). In contrast, self-produced movements of the limbs engender temporal activations within (Astafiev et al., 2004) or in close proximity to (Peelen and Downing, 2005b) EBA, suggesting a functional link between motor systems and visual body representations. Furthermore, rTMS over vPMc impairs the visual discrimination of static (Urgesi et al., 2007) and dynamic displays of moving body parts (Pobric and Hamilton, 2006), suggesting that motor representations are necessary for visual discrimination of others' actions. Our study significantly expands previous research by investigating what aspects of visual body stimuli are processed in different body-related areas of the visual and sensorimotor cortices. Our results show that $\mathrm{vPMc}$ is required for the configural processing of observed human body postures. This suggests that $\mathrm{vPMc}$ provides an embodied representation of whole-body configurations and that we may use our own body representations to represent the body of others.

The possible role of motor representations in the configural perceptual processing of body postures is suggested by the absence of body inversion effect in children with autism spectrum disorder (Reed et al., 2006b), a clinical condition in which defective neural activity in vPMc during gesture observation has been found (Dapretto et al., 2006). We therefore posit that altered motor representations of observed actions may impair configural perceptual processing of body postures. In a similar vein, motor representations may be sufficient for configural body processing even in the absence of on-line, proprioceptive representation of the observer' body as indicated by the preserved body inversion effect in a patient with complete somatic deafferentation, but with intact efferent motor signals (Bosbach et al., 2006). Whether configural processing of body and face stimuli is hard-wired in the brain or develops as a consequence of the experience we have with visual processing other individuals' bodies and faces (Bukach et al., 2006) is still a matter of debate. Our finding that vPMc is crucial for configural processing of body postures would suggest that the experience we have with our own body and movements may have a major influence on cognitive strategies and neural processing implemented during visual analysis of human bodies.

Body inversion effect was also reduced by stimulation of right SPL. Neuropsychological studies indicate that cerebral lesions, particularly when centered on the right parietal lobe, may ensue in specific alterations of the spatial representation of one's own body (Halligan et al., 1995; Aglioti et al., 1996; Berlucchi and Aglioti, 1997; Guariglia et al., 2002). Neuroimaging studies have documented the activation of right SPL during the task of mental transformation of body in space (Bonda et al., 1995) as well as during action observation (Buccino et al., 2001; Calvo-Merino et al., 2005). Furthermore, neuroimaging studies show that SPL response to upright moving bodies is higher than to inverted bodies (Thompson et al., 2005). In a similar vein, the absence of body inversion effect after stimulation of SPL may indicate its involvement in the configural processing of human bodies. However, accuracy in matching upright postures was significantly lower after vPMc than V1 stimulation. No difference with respect to V1 was found after SPL stimulation. Therefore, vPMc may be a more strongly configural processing area than SPL. Although it is plausible that the stimulation delay used in the present study was not particularly adept for detecting the possible involvement of parietal areas in the configural processing of body stimuli, only future studies will clarify this issue.

\section{Visual processing of human bodies}

Neuroimaging studies report that visual perception of still and moving bodies selectively activates specific regions of temporoparietal cortex (Downing et al., 2001; Grossman and Blake, 2002; Peelen et al., 2006). However, little is known about the type of body processing performed in these areas. By using functional magnetic resonance imaging, Thompson et al. (2005) found higher activation during whole-body observation compared with observation of fragmented body parts in bilateral superior temporal sulcus areas and in right lateral temporal cortex close to the EBA location. Moreover, they showed stronger activity in lateral temporal cortex for upright versus inverted biological motion stimuli. This would suggest the involvement of superior temporal sulcus area and of EBA in the configural processing of body movements. However, given the technique used in the study, it was not possible to establish whether changes of neural activity reflected a causal or merely incidental involvement of these areas. It is worth noting that rTMS of superior temporal sulcus areas impairs perception of upright but not inverted biological motion stimuli (Grossman et al., 2005), thus suggesting that this area plays a crucial role in configural processing of body movements. Superior temporal activations, however, do not occur during observation of static bodies (Grossman and Blake, 2002), whereas EBA responds to visual presentation of moving as well as still bodies (Downing et al., 2001). Our study indicates that EBA analyzes bodies by coding the details of human body parts instead of whole-body configurations. This kind of body processing becomes essential when processing the whole-body configuration has been disrupted by inversion. Under naturalistic conditions perceiving the details of human body parts may be crucial for monitoring the body-derived identity of others (Urgesi et al., $2004,2007)$. Indeed, the configurations of human bodies in space 
change dramatically during action. Furthermore, the bodies of different individuals may assume exactly the same posture and perform the same movements, so that their configural properties exactly match. In those conditions, the local details of human body parts may be important for person identification.

\section{Two specialized systems for the visual processing of human bodies}

Inversion of face and body stimuli impairs face and body perception, thus suggesting that these stimuli are processed as whole units. Despite this similarity, configural processing of faces and bodies may be different. Faces may be processed as undifferentiated wholes (Maurer et al., 2002), whereas configural processing of bodies seems to be based on the spatial relationships among body parts in the context of the whole-body space (Reed et al., 2006a). Configural processing of faces seems to be subserved by the fusiform face area, whereas the local processing of body-part details may involve activity in occipito-temporal face-selective areas (Haxby et al., 2000).

The present study shows that configural processing of bodies requires sensorimotor representations rather than visual representation in the extrastriate visual cortex, which seems to be involved in local body processing. We propose that the human brain contains two dissociable and independent routes that are specialized for processing human bodies (Fig. $5 B$ ). One route may process the whole body in a configural manner and involve dorsal system areas, such as vPMc. This suggests that configural processing of bodies may imply the embodiment of observed postures onto the observer's sensorimotor representations. A second route may be specialized for processing local features of the body, such as body parts and body form. EBA, which is part of the ventral system and may be specialized for the processing of human body form and features, may be crucial for local processing of body part details. Future studies are needed to better understand the relative contribution of lateral temporal (Downing et al., 2001) and fusiform body-selective areas (Peelen and Downing, 2005a) to body perception.

\section{References}

Aglioti S, Smania N, Manfredi M, Berlucchi G (1996) Disownership of left hand and objects related to it in a patient with right brain damage. NeuroReport 8:293-296.

Amassian VE, Cracco RQ, Maccabee PJ, Cracco JB (2002) Visual system. In: Handbook of transcranial magnetic stimulation (Pascual-Leone A, Davey NJ, Rothwell JC, Wasserman EM, Puri BK, eds), pp 323-334. London: Oxford UP.

Astafiev SV, Stanley CM, Shulman GL, Corbetta M (2004) Extrastriate body area in human occipital cortex responds to the performance of motor actions. Nat Neurosci 7:542-548.

Berlucchi G, Aglioti S (1997) The body in the brain: neural bases of corporeal awareness. Trends Neurosci 20:560-564.

Bonda E, Petrides M, Frey S, Evans A (1995) Neural correlates of mental transformations of the body-in-space. Proc Natl Acad Sci USA 92:11180-11184.

Bosbach S, Knoblich G, Reed CL, Cole J, Prinz W (2006) Body inversion effect without body sense: insights from deafferentation. Neuropsychologia 44:2950-2958.

Briggs GG, Nebes RD (1975) Patterns of hand preference in a student population. Cortex 11:230-238.

Buccino G, Binkofski F, Fink GR, Fadiga L, Fogassi L, Gallese V, Seitz RJ, Zilles K, Rizzolatti G, Freund HJ (2001) Action observation activates premotor and parietal areas in a somatotopic manner: an fMRI study. Eur J Neurosci 13:400-404.

Bukach CM, Gauthier I, Tarr MJ (2006) Beyond faces and modularity: the power of an expertise framework. Trends Cogn Sci 10:159-166.

Calvo-Merino B, Glaser DE, Grezes J, Passingham RE, Haggard P (2005)
Action observation and acquired motor skills: an FMRI study with expert dancers. Cereb Cortex 15:1243-1249.

Clarke TJ, Bradshaw MF, Field DT, Hampson SE, Rose D (2005) The perception of emotion from body movement in point-light displays of interpersonal dialogue. Perception 34:1171-1180.

Dapretto M, Davies MS, Pfeifer JH, Scott AA, Sigman M, Bookheimer SY, Iacoboni M (2006) Understanding emotions in others: mirror neuron dysfunction in children with autism spectrum disorders. Nat Neurosci 9:28-30.

Dittrich WH, Troscianko T, Lea SE, Morgan D (1996) Perception of emotion from dynamic point-light displays represented in dance. Perception 25:727-738.

Downing PE, Jiang Y, Shuman M, Kanwisher N (2001) A cortical area selective for visual processing of the human body. Science 293:2470-2473.

Grezes J, Armony JL, Rowe J, Passingham RE (2003) Activations related to "mirror" and "canonical" neurones in the human brain: an fMRI study. NeuroImage 18:928-937.

Grossman E, Blake R (2002) Brain areas active during visual perception of biological motion. Neuron 35:1167-1175.

Grossman ED, Battelli L, Pascual-Leone A (2005) Repetitive TMS over posterior STS disrupts perception of biological motion. Vision Res 45:2847-2853.

Guariglia C, Piccardi L, Puglisi Allegra MC, Traballesi M (2002) Is autotopoagnosia real? EC says yes. A case study. Neuropsychologia 40:1744-1749.

Halligan PW, Marshall JC, Wade DT (1995) Unilateral somatoparaphrenia after right hemisphere stroke: a case description. Cortex 31:173-182.

Haxby JV, Hoffman EA, Gobbini MI (2000) The distributed human neural system for face perception. Trends Cogn Sci 4:223-233.

Kanwisher N, McDermott J, Chun MM (1997) The fusiform face area: a module in human extrastriate cortex specialized for face perception. J Neurosci 17:4302-4311.

Luber B, Kinnunen LH, Rakitin BC, Ellsasser R, Stern Y, Lisanby SH (2007) Facilitation of performance in a working memory task with rTMS stimulation of the precuneus: frequency- and time-dependent effects. Brain Res 1128:120-129.

Maurer D, Grand RL, Mondloch CJ (2002) The many faces of configural processing. Trends Cogn Sci 6:255-260.

Oliveri M, Turriziani P, Carlesimo GA, Koch G, Tomaiuolo F, Panella M, Caltagirone C (2001) Parieto-frontal interactions in visual-object and visual-spatial working memory: evidence from transcranial magnetic stimulation. Cereb Cortex 11:606-618.

Pavlova M, Sokolov A (2000) Orientation specificity in biological motion perception. Percept Psychophys 62:889-899.

Peelen MV, Downing PE (2005a) Selectivity for the human body in the fusiform gyrus. J Neurophysiol 93:603-608.

Peelen MV, Downing PE (2005b) Is the extrastriate body area involved in motor actions? Nat Neurosci 8:125.

Peelen MV, Wiggett AJ, Downing PE (2006) Patterns of fMRI activity dissociate overlapping functional brain areas that respond to biological motion. Neuron 49:815-822.

Pobric G, Hamilton AF (2006) Action understanding requires the left inferior frontal cortex. Curr Biol 16:524-529.

Postle BR, Ferrarelli F, Hamidi M, Feredoes E, Massimini M, Peterson M, Alexander A, Tononi G (2006) Repetitive transcranial magnetic stimulation dissociates working memory manipulation from retention functions in the prefrontal, but not posterior parietal, cortex. J Cogn Neurosci 18:1712-1722.

Ranganath C, D'Esposito M (2005) Directing the mind's eye: prefrontal, inferior and medial temporal mechanisms for visual working memory. Curr Opin Neurobiol 15:175-182.

Reed CL, Farah MJ (1995) The psychological reality of the body schema: a test with normal participants. J Exp Psychol Hum Percept Perform 21:334-343.

Reed CL, Stone VE, Bozova S, Tanaka J (2003) The body-inversion effect. Psychol Sci 14:302-308.

Reed CL, Stone VE, Grubb JD, McGoldrick JE (2006a) Turning configural processing upside down: part and whole body postures. J Exp Psychol Hum Percept Perform 32:73-87.

Reed CL, Beall PM, Stone VE, Kopelioff L, Pulham DJ, Hepburn SL (2006b) Brief report: Perception of body posture-what individuals with autism spectrum disorder might be missing. J Autism Dev Disord, in press. 
Rizzolatti G, Craighero L (2004) The mirror-neuron system. Annu Rev Neurosci 27:169-192.

Sawaki L, Okita T, Fujiwara M, Mizuno K (1999) Specific and non-specific effects of transcranial magnetic stimulation on simple and go/no-go reaction time. Exp Brain Res 127:402-408.

Stekelenburg JJ, de Gelder B (2004) The neural correlates of perceiving human bodies: an ERP study on the body-inversion effect. NeuroReport 15:777-780.

Talairach J, Tournoux P (1988) Co-planar stereotaxic atlas of the human brain Stuttgart, Germany: Thieme.

Terao Y, Ugawa Y, Suzuki M, Sakai K, Hanajima R, et al (1997) Shortening of simple reaction time by peripheral electrical and submotor-threshold magnetic cortical stimulation. Exp Brain Res 115:541-545.

Thompson JC, Clarke M, Stewart T, Puce A (2005) Configural processing of biological motion in human superior temporal sulcus. J Neurosci 25:9059-9066.

Urgesi C, Berlucchi G, Aglioti SM (2004) Magnetic stimulation of extrastri- ate body area impairs visual processing of nonfacial body parts. Curr Biol $14: 2130-2134$

Urgesi C, Moro V, Candidi M, Aglioti SM (2006) Mapping implied body actions in the human motor system. J Neurosci 26:7942-7949.

Urgesi C, Candidi M, Ionta S, Aglioti SM (2007) Representation of body identity and body actions in extrastriate body area and ventral premotor cortex. Nat Neurosci 10:30-31.

Wasserman EM (1998) Risk and safety of repetitive transcranial magnetic stimulation: report and suggested guidelines from the International Workshop on the Safety of Repetitive Transcranial Magnetic Stimulation, June 5-7, 1996. Electroencephalogr Clin Neurophysiol 108:1-16.

Wilson M, Knoblich G (2005) The case for motor involvement in perceiving conspecifics. Psychol Bull 131:460-473.

Yin RK (1969) Looking at upside-down faces. J Exp Psychol 81:141-145.

Yovel G, Kanwisher N (2005) The neural basis of the behavioral faceinversion effect. Curr Biol 15:2256-2262. 